\title{
Hypertension, Autonomic Storm, Increased Counter Regulatory Hormones and Suppressed Insulin in Acute Myocarditis in Scorpion Envenoming Syndrome
}

\author{
Kari Radha Krishna Murthy \\ Department of Physiology, Santhiram General Hospital \& Santhiram Medical College, Kurnool, India \\ Email: kradhakrishnamurthy@yahoo.com
}

Received 9 March 2014; revised 13 April 2014; accepted 21 April 2014

Copyright (C) 2014 by author and Scientific Research Publishing Inc. This work is licensed under the Creative Commons Attribution International License (CC BY). http://creativecommons.org/licenses/by/4.0/

\section{Abstract}

Death due to scorpion envenoming syndrome is a common event in many of the tropical and nontropical counties. Initial transient hypertension is commonly observed in scorpion sting victims. Scorpion envenoming causes autonomic storm resulting in initial transient hypertension followed by hypotension, cold clammy skin, hypothermia, cardiovascular disturbances, acute myocarditis, sarcolemmal defects, pulmonary oedema, acute pancreatitis, disseminated intravascular coagulation (DIC), Adult respiratory distress syndrome (ARDS), and many other clinical manifestations. All these manifestations could be due to sudden increase in catecholamines, angiotensin II, glucagon, Cortisol and either due to suppressed insulin secretion or insulin resistance and death. The sudden increase of metabolic $A$ in counter-regulatory hormones along with either suppressed insulin secretion/or insulin resistance results in glycogenolysis in liver, cardiac and skeletal muscles causing hyperglycemia and a sudden increase in free fatty acid levels. Free Fatty Acids increase the susceptibility of the ventricles to the disorganized electrical behavior, inhibit cardiac sarcolemmal $\mathrm{Na}^{+}-\mathrm{K}^{+}$ATPase activity, increase the tendency to intravascular thrombus, increase myocardial oxygen consumption, interfere with tropomyosin-troponin activation of Actin-Myosin coupling, show detergent effects on cell membranes and they could alter the stabilization of lysosomal membranes and probably become toxic to the myocardium. Based on our animal experiments in which insulin administration reversed the metabolic and ECG changes induced by scorpion envenoming and treating the poisonous scorpion sting victims with insulin, we consider that insulin has a primary metabolic role in preventing, counter-acting and reversing all the deleterious effects of FFA by inhibiting the catecholamine induced by lipolysis, and increasing intra-cellular $\mathrm{K}^{+}$, facilitating glucose transport to the myocardium and glucose metabolism through different pathways. Administration of insulin-glucose infusion to scorpion sting victims appears to be the physiological basis for the control of the metabolic response when that has become a de- 
terminant to survival. Treatment using continuous infusion of regular crystalline insulin should be given at the rate of $0.3 \mathrm{U} / \mathrm{g}$ glucose and glucose at the rate of $0.1 \mathrm{~g} / \mathrm{kg}$ body weight $/ \mathrm{hour}$, for 48 72 hours, with supplementation of potassium as needed and maintenance of fluid, electrolytes and acid-base balance.

\section{Keywords}

\section{Hypertension, Autonomic Storm, Angiotensin II, Adult Respiratory Distress Syndrome (ARDS), Multi-System Organ Failure (MSOF), Insulin-Glucose Infusion}

\section{Introduction}

Death due to scorpion envenoming is a common problem especially in the developing countries all over the world. Scorpion envenoming syndrome could be due to autonomic storm resulting in acute myocarditis \& hypotension [1]-[35], initial transient hypertension-increased systemic blood pressure, electrocardiographic changes, arrhythmias, conduction defects, ischemia and infarction like patterns; sudden fall in arterial blood pressure [1][5] [16]-[27], cardiovascular disturbances [14]-[26], acute pancreatitis [25] [33], Disseminated Intravascular Coagulation (DIC) [23], Adult Respiratory Distress Syndrome (ARDS) [28] [30], many other life threatening clinical manifestations and death [31]. All these manifestations could be due to alterations in the hormonal mileu-massive release of catecholamines [1]-[10] [12]-[15] [31]-[33], angiotensin II [19], glucagon, glucocorticoid secretions [17] and either suppressed insulin secretion [17] [22]-[24] [26] [29] or hyper-insulinemia [17] [22]-[24] [26] [29] with hyperglycemia [6]-[8] [17] [21]-[24] [26] [29]-[35], lipolysis (breakdown of triglycerides) with sudden increase in free fatty acid levels [6]-[8] [17] [18] [21]-[24] [26] [29] and death.

Scorpions of Buthidae family are toxic and cause death. Scorpion sting is a major public health problem in tropical and subtropical areas of the world. Nearly 1000 species of scorpions belonging to 6 families have been described. Species of scorpions that belong to Buthidae family produce neurotoxic secretions that are toxic and cause death to humans. Among the most toxic species are the Mesobuthus tamulus of India, the Leiurus quinquestriatus of North Africa and the Near East, the Tityus serrulatus of Brazil, the Leiurus quinquestriatus of Israel, the Centruroides suffuses of Mexico, the Tityus triniatis of Trinidad, the Leiurus quinquestriatus of Tunisia and many more. Scorpion stings are also common in Southwestern areas of United States of America, Mexico, Algeria, Saudi Arabia, Brazil, China, India, Central Africa and South Africa. Despite zoological differences among the various poisonous species under Family Buthidae, symptomatology in humans following envenoming is quite similar [36]-[38].

The physiological basis of various patho-physiological mechanisms as a consequence of autonomic storm in scorpion envenoming syndrome and its reversal of the actions of counter-regulatory hormones by administration of insulin in the experimental animals and scorpion sting victims [19] [22]-[24] [26] [27] [30] are reviewed under the following headings.

\subsection{Hypertension in Scorpion Sting Victims with Elevated Cardiac Enzymes}

Hypertension is commonly observed in scorpion sting children. Severe or significant hypertension was observed in 77\% of victims [1]-[5] [31]-[35]. Sofer and Gueron reported hypertension (ranging between 172/125 mmHg to $150 / 120 \mathrm{mmHg}$ ) in scorpion sting children (age 1 year to 2.8 years) with myocardial infarction like pattern, interstial pulmonary oedema, elevated creatine phosphokinase (CPK) levels (range 277 to $3000 \mathrm{U} /$ liter), Serum Glutamine Oxaloacetic Transaminase levels (SGOT) (range 64 - 240 U/liter) and elevation of creatine phosphokinase isoenzyme (CPK-MB) fraction elevated to 110 U/liter) [37]. Myocarditis, convulsions, brain oedema, shock and respiratory distress were encountered in scorpion sting children from Jerusalem [39]-[43].

Gueron, Margulis and Sofer from Israel reported severe hypertension (ranging between 180/120 $\mathrm{mmHg}$ to 150/103 mmHg), elevated SGOT levels (range 40 - 57 Units), increased CPK levels (range 238 - 524 U/liter), MB-PK ratio (\%) (Range 6.8\% - 20\%) in children (age 0.5 year to 14 years). Abnormal echocardiograms were observed. Poorly contracting wall motion (global) with decreased systolic left ventricular performance and normal wall thickness was present in these scorpion sting children [39]. 
Arterial hypertension, tremors, hyperglycemia, hypothermia and hypotension was observed due to severe scorpion envenomation in children caused by Tityus bahiensis and Tityus serrulatus from Brazil [31].

Sofer et al. observed hypertension in 21 out of 27 in children with myocardial injury due to Leiurus quinquestriatus envenoming from Israel. Many of these children had enzymatic myocardial involvement characterized by high total CPK level, elevated CK-MB level, CK-MB/CPK ratio exceeding 6\% and ECG changes consistent with myocardial damage [40].

\subsection{Elevated Plasma Nor-Epinephrine Levels}

Plasma nor-epinephrine levels were elevated on admission $(1279 \mathrm{pg} / \mathrm{ml})$ in children stung by Tityus zulianus from Venezuela [41]. The seriousness of scorpion envenomation could be essentially from left cardiac dysfunction with pulmonary oedema and/or a state of shock. Adrenergic myocarditis, toxic myocarditis and myocardial ischemia are the three mechanisms that explain the cardiac dysfunction. Myocardial ischemia is not only due to the release of catecholamines but also the effect of the cytokines and/or neuropeptides $y$ on the coronary vessels. The cardiac damage can be due or enhanced by the depressive effect of the cytokines on the myocardial cells. The frequently observed hyperglycemia only enhances the state of the already damaged myocardium [42].

\subsection{Catecholamine Metabolites Excretion}

Sofer and Gueron [1]-[5] reported the catecholamine metabolites excretion in 12 scorpion sting victims. Abnormal serum level and increased catecholamine metabolite urine excretion was also reported from scorpion sting patient.

\subsection{Elevated Circulating Levels of Catecholamines and Rennin Angiotensin}

Elevated circulating levels of catecholamines and rennin angiotensin had been observed in clinical and experimental envenomation [5] [9] [19].

\subsection{A Conspicuous Increase in the Arterial Blood Adrenaline Content and a Marked Rise in the Arterial Blood Pressure (Figure 1, Figure 2)}

A conspicuous increase in the arterial blood adrenaline content and a marked rise in the arterial blood pressure were observed upon injection of Middle East scorpion (Leiurus quinquestriatus) venom in dogs [1]-[5] [6]-[10] [19]. Administration of South American Tityus serrulatus venom in rats caused a rise in the arterial blood pressure and an increase in catecholamine level. A toxin purified from the crude venom of the North African scorpion (Leiurus quinquestriatus) induced the release of catecholamines. The clinical picture of the patients stung by scorpion from Middle East seems to bear a certain relationship to the level of adrenaline in the plasma [9] [10].

Intra Venous administration of crude venom of the scorpion (B. tamulus) in rats produced a vasopressor response. This pressor response is mediated through an indirect mechanism of catecholamine release from peripheral sites including the adrenal medulla [9] [10].

\subsection{Initial Transient Hypertension Followed by Hypotension in the Experimental Scorpion Envenoming}

Kymographic recording of arterial blood pressure after administration of scorpion venom is shown in Figure 1. Initial transient hypertension followed by hypotension is observed in our experimental animals. Initial transient hypertension followed by hypotension in experimental dogs could be due to massive discharge of catecholamines [5] [9] [19].

\subsection{Elevation of Blood Pressure and Plasma Renin Levels}

Stimulation of the sympathetic nervous system [10] can increase the renin output [5]. La Grange demonstrated elevation of blood pressure and plasma renin levels by Centruroides sculpturatus and Leiurius quinquestriatus venoms and proposed that the renin-angiotensin system participates in the hypertensive response to scorpion venom along with the increased catecholamines [9] [34]. Other workers using B. minax and B. tamulus venoms 


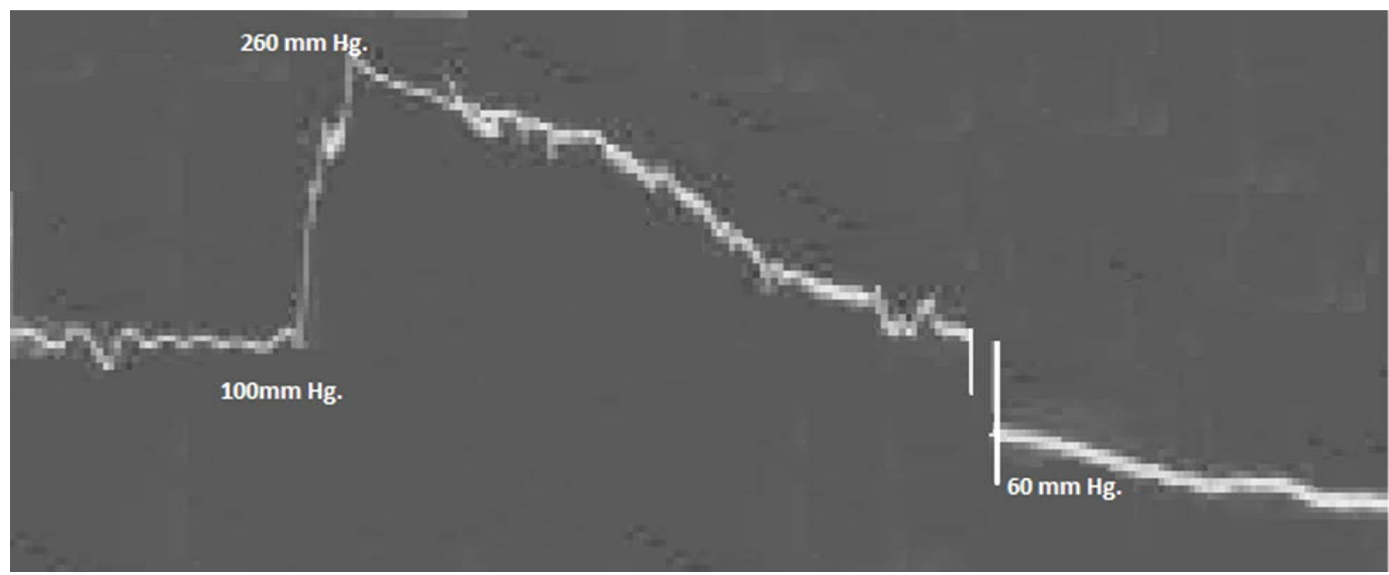

Figure 1. Kymographic Recording of Mean arterial blood pressure in the experimental dog after injection of scorpion venom.

\section{SCORPION ENVENOMING}

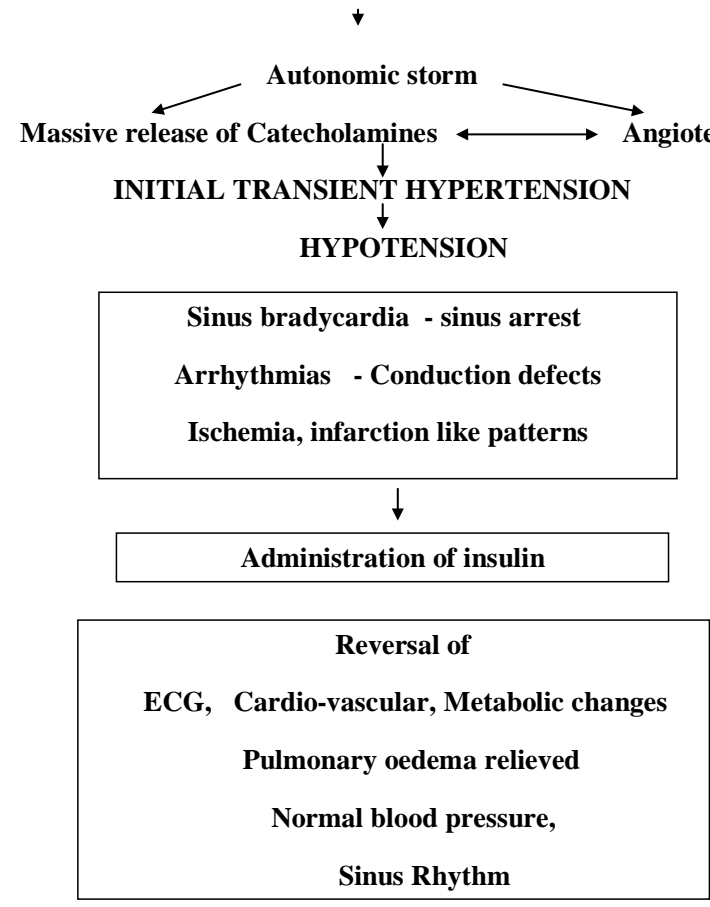

Figure 2. Mechanism of changes in the Mean Arterial Blood Pressure due to scorpion venom \& its reversal by insulin administration.

were able to block the hypertensive action using alpha blocking agent phenoxybenzamine. However, in the hands of La Grange alpha blocking did not prevent the hypertensive effect of the venom [9].

\subsection{Inotropic State Due to Increase in the Level of Circulatory Catecholamines}

Tarasiuk et al. [43] observed in their experimental dogs an inotropic state immediately following intravenous injection of $0.05 \mathrm{mg} \cdot \mathrm{kg}^{-1}$ venom from scorpion Leiurus quinquestriatus. The inotropic state has been ascribed to the increase in the level of circulatory catecholamines and produced the following changes.

1) Blood pressure: Baseline Blood pressure was $98 \mathrm{mmHg}$. Blood pressure increased to $177 \mathrm{mmHg}$ at $15 \mathrm{~min}$, $168 \mathrm{mmHg}$ at $30 \mathrm{~min}$ after venom injection. The Blood pressure dropped to $88 \mathrm{mmHg}$ at $90 \mathrm{~min}$ and $65 \mathrm{mmHg}$ 
at $120 \mathrm{mmHg}$, decreased to $63 \%$ below baseline value following envenomation.

2) Mean Circulatory Pressure (MCP): MCP provides the best estimate of the upstream pressure driving blood to the heart, because it is relatively independent of cardiac output. At baseline, MCP was 6 - 8 torr. Five minutes following venom injection, MCP increased by 300\% and remained elevated for 60 min. At 120 min, MCP returned to baseline values.

3) Cardiac output (CO): Baseline Cardiac output $\left(\mathrm{L} \cdot \mathrm{min}^{-1}\right)$ was 2.9. Fifteen minutes following venom injection, CO increased to $250 \%$ following envenomation. CO dropped to $40 \%$ and $35 \%$ below baseline at 90 and 120 min following venom injection.

4) Systemic Vascular Resistance (SVR): SVR increased by 35\% by 90 min after venom injection.

5) Pulmonary Vascular Resistance (PVR): PVR increased to a maximal value of 53\% above baseline at 120 min following venom injection.

6) Arterial Critical Closing Pressure $\left(\mathrm{P}_{\text {crit }}\right)$ : It is the minimal pressure required to keep the blood vessel open. $\mathrm{P}_{\text {crit }}$ was $32 \mathrm{mmHg}$ before venom injection. It increased to $102 \mathrm{mmHg}$ at $15 \mathrm{~min}, 107 \mathrm{mmHg}$ at $30 \mathrm{~min}$, and became $38 \mathrm{mmHg}$ at $90 \mathrm{~min}$ and $28 \mathrm{mmHg}$ at $120 \mathrm{~min}$ following envenomation [43].

7) Redistribution of blood flow: The increase in MCP immediately following venom injection is probably due to Sympathoadrenal response known to occur following scorpion envenoming. This effect is mediated either by vasoconstriction of peripheral venous reservoirs or by shift of blood from unstressed to stressed vascular compartments. An increase in circulating catecholamines can induce reduction of vascular capacitance, leading to redistribution of blood flow to organs, and can induce an increase in Right Atrial Pressure (RAP). According to Frank-Starling mechanism, elevation of RAP will contribute to an increase in cardiac output [43].

\subsection{Other Underlying Mechanism That Explain the Hemodynamic Changes}

Another underlying mechanism that explains the haemodynamic changes may involve activation and release of cytokines and other pro-inflammatory substances, causing pathophysiological cascades similar to those seen in endotoxic shock.

1) “Systemic Inflammatory Response Syndrome” (SIRS) following scorpion sting

Elevated serum interleukin-6 is reported in patients admitted with "Systemic Inflammatory Response Syndrome” (SIRS) following scorpion sting [36].

2) Scorpion venom-neuropeptides-effect of endothelin release

Scorpion venom is composed primarily of neuropeptides, which target ion channels. Since the movement of sodium ions $\left(\mathrm{Na}^{+}\right)$, Potassium ions $\left(\mathrm{K}^{+}\right)$, chloride ions $\left(\mathrm{Cl}^{-}\right)$and Calcium ions $\left(\mathrm{Ca}^{2+}\right)$ in and out of the cells through ion channels is absolutely essential in neural and muscular function, these channels act as primary targets for the offending scorpion venom. Neurotoxins present in the scorpion venom affect the gating mechanisms of excitable membrane. Long toxins (60 - 70 amino acids) interfere with sodium channels, causing cell destruction by the influx of $\mathrm{Na}^{+}$and $\mathrm{Ca}^{2+}$ ions into the cell. Short toxins (31 - 37 amino acids) modify the potassium channel's function and may specially block $\mathrm{Ca}^{2+}$ activated $\mathrm{L}^{+}$channels. These effects on vascular endothelial cells may exert different reactions on different organs. All these responses may resemble the effect of endothelin release [36] [43].

3) Other probable mechanisms that could explain "Hypertension"

It is possible that the scorpion venom, through delaying the inactivation of the fast $\mathrm{Na}^{+}$channels and blocking the $\mathrm{Ca}^{2+}$ activated $\mathrm{K}^{+}$channels, would enhance the rate of afferent discharge along the sensory nerves causing increased neurotransmitter release within the central nervous system, augmented reflexes, autonomic and somatic effects. The nerve terminals are likely the primary site of venom action. The excitatory pre-synaptic action of scorpion venom is due to an action at the relatively exposed regions of the nerves at their endings closer to the synaptic regions. A selective action of scorpion venom on the sympathetic and parasympathetic centers of the medulla would explain most of the effects of the venom. Stimulation of the hypothalamus by scorpion venom could explain more adequately the pathophysiology of scorpion envenomation. The disturbance in the integrative function of the hypothalamic centers can explain such symptoms as shivering, piloerection, increased body temperature, hyperirritability, and leucocytosis. Tityus toxin caused nearly eight fold increase in transmitter release after the preparation was incubated. The enhancement of transmitter release is likely due to the toxin-induced potentiation of the duration of the action potential. The nerve fiber apparently remains sensitized for a long period of time; the response to a second stimulus after a previous exposure to scorpion venom is probably amplified several fold [8]. 
4) Mesobuthus tamulus concanesis, Pocock prolongs repolarization and refractory time of the compound action potential

Indian red scorpion (Mesobuthus tamulus concanesis, Pocock; formerly known as Buthus tamulus; MBT) venom prolonged the repolarization and refractory time of the compound action potential by involving calciumdependent mechanism through both $\mathrm{Ca}^{2+}$ sensitive and $\mathrm{Ca}^{2+}$ insensitive mechanisms. The $\mathrm{Ca}^{2+}$ influx occurred via L-type of calcium channels [44] [45].

a) Significant prolongation of the repolarization time

Significant prolongation of the repolarization time was seen with $0.6 \mathrm{ug} / \mathrm{ml}$ of MBT venom. The maximal prolongation of the repolarization time, nearly 100 times the duration of the control was seen with $6 \mathrm{ug} / \mathrm{ml}$ of the venom [44].

b) Significant increases in refractory period

MBT venom $(0.6 \mathrm{ug} / \mathrm{ml})$ increased the refractory period by 2.5 times the control response. The maximal prolongation of the refractory period (225 times the duration of the control) was seen with $6 \mathrm{ug} / \mathrm{ml}$ of the venom [44].

c) The autonomic storm after scorpion envenoming may be due to neuronal changes

The repolarization of the compound action potential is due to the inactivation of $\mathrm{Na}^{+}$permeability (channels) and activation of $\mathrm{K}^{+}$permeability. These events occur within 2 - 3 msec and restore the membrane potential to its original state under normal conditions. The refractoriness depends upon the restoration of the membrane potential by inactivation of $\mathrm{Na}^{+}$system. The toxins from other poisonous species of scorpions (Leiurus quinquestriatus and Tityus serrulatus) prolonged the duration of action potential by slowing the $\mathrm{Na}^{+}$inactivation process. MBT venom increases the neuronal excitability. The autonomic storm after scorpion envenoming may be due to such neuronal changes [44].

\subsection{Plasma Angiotensin Levels Were Elevated after Scorpion Venom Injection in Dogs as Well as Rabbits}

Following the injection of Mesobuthus tamulus concanesis, Pocock venom, plasma angiotensin levels were elevated in dogs as well as rabbits [19].

\subsection{Administration of Insulin Reduced Plasma Angiotensin Levels in Dogs as Well as Rabbits}

Administration of insulin reduced these levels in dogs. Administration of Insulin, Tolazoline (alpha blocker) and sodium bicarbonate reduced the plasma angiotensin levels, drastically [19].

\subsection{Low Concentrations of Scorpion Venom Produce Sustained Hypertensive Response and an Increase in Plasma Renin Activity}

Relatively low concentrations of scorpion venom (Leiurus quinquestiatus) are capable of producing sustained hypertensive response and an increase in plasma renin activity. Venom from scorpion, Centruroides sculpturatus and Leiurus quinquestiatus elevated plasma renin activity. The determination of plasma renin activity has been widely adapted to evaluate the renin angiotensin generated per $\mathrm{ml}$ of plasma per unit time at physiological temperature and serves as an index of the renin activity [5].

\subsection{Highly Abnormal Renin and Aldosterone Levels in Scorpion Sting Child}

Renin and aldosterone levels evaluated in a 2-year old patient showed highly abnormal values: Renin-35 nano/ $\mathrm{ml} /$ hour (normal 0.2 - 2.8) and aldosterone $1000 \mathrm{pg} / \mathrm{ml}$ (normal 15 - $150 \mathrm{pg} / \mathrm{ml}$ ) [1]-[5] [34] [35].

\section{ECG Changes in Acute Myocarditis Due to Scorpion Envenoming}

The ECG is the most important laboratory aid in the diagnosis of acute myocarditis. The cardiac effects in the experimental animals were comparable to the changes observed in humans accidentally stung by scorpion.

1) Abnormal ECG changes after envenoming

Scorpion sting myocardial toxicity is more common than neurotoxicity and often is the cause of death. Acute 
myocarditis is frequently unrecognized because of the severity of the associated condition [20].

The scorpion venom is a powerful arrhythmogenic agent by virtue of stimulating autonomic sympathetic system and adrenal glands. The common immediate arrhythmias are sinus arrest or sinus bradycardia with different escape rhythms (for example: Junctional rhythm), premature ventricular tachycardia. Each one of these has been recorded by us. Further, the arrhythmias were present before the full haemodynamic venom induced effects [20].

Many abnormal ECG changes were observed after envenoming. These include early myocardial infarction like pattern with ST segment elevation, ST segment depression, Presence of Q waves. These abnormalities were observed within a fraction of a minute after administration of the scorpion venom. In addition to these changes, there were: Rhythm disturbances such as AV disassociation, SA Block, Atrial tachycardia and Ventricular tachycardia, Bundle branch block and Pericarditis like patterns [16]-[30] [45].

2) a) ECG changes in our experimental animals

The ECG was recorded continuously in the experimental animals after intravenous injection of $4 \mathrm{mg} / \mathrm{kg}$ venom from scorpion (Mesobuthus tamulus Concanesis, Pocock). Several abnormalities were recorded in the ECG tracings pertaining to changes in the configuration of complexes, conduction defects at various levels and arrthymias of several types. The abnormalities included sinus bradycardia, sinus tachycardia, prolonged PR interval, Short PR interval, and abnormal Q wave, narrow QRS, broad QRS of intra-ventricular conduction defect type, Bundle Branch Block pattern, flat T wave, inverted T wave, ST segment elevation and ST segment depression, myocardial infarction like pattern. ECG tracings also showed change of axis, electrical alternans, First degree heart block, Junctional rhythm, atrial and ventricular fibrillation. We have recorded myocardial infarction like pattern, Junctional rhythm and electrical alternans hitherto unreported in experimental myocarditis but reported in human victims in 1986! Change of axis in the ECG tracings noticed by us has not been reported either in the experimental studies or in patients affected by scorpion stings. We claim to have recorded almost all ECG changes (in the experimental studies) noticed in humans affected by scorpion stings [20].

b) ECG changes in our experimental rabbits after scorpion envenoming

Hyperacute injury pattern with ST elevation, Ventricular tachycardia, Sinus tachycardia with ST-T changes, Acute infarction, Sinus arrest, Infarction like pattern, Runs of ventricular premature beats, Multi-focal ventricular premature beats [6]-[30] [45].

c) ECG abnormalities in our experimental animals with "acute myocarditis”

Electrocardiographic changes reported in the experimentally induced myocarditis by the poisonous scorpion venom injection have been: 1) Sinus tachycardia; 2) Sinus bradycardia; 3) T inversion; 4) ST elevation; 5) ST depression; 6) AV conduction defects with sinus arrest; 7) Ventricular premature contraction; 8) T wave configuration changes; 9) Prolonged PR; 10) Short PR; 11) ST depression; 12) Narrow QRS; 13) Wide QRS and 14) Extra systoles [16]-[30] [45].

We have reported myocardial infarction like pattern with ST elevation, Ventricular tachycardia, Sinus tachycardia with ST-T changes, acute infarction, Sinus arrest, Infarction like pattern, runs of ventricular premature beats, Multi-focal ventricular premature beats [16]-[30] [45].

d) ECG changes observed in our experimental dogs [16]-[30] [45]

Myocardial infarction like pattern

Escape Beats \& Escape Rhythms: Junction rhythm, Junction escape, ventricular escape

Premature Systoles \& Para systoles: APC, Bigeminy, Junctional extra systoles, VPC

Paroxysmal \& Non-paroxysmal Tachycardia: Nodal tachycardia, Ventricular tachycardia

Fibrillation \& Flutter: Ventricular fibrillation

Conduction Block: S-A block, A-V dissociation, Bundle branch block, Wide QRS

Other disturbances in ECG: Sinus tachycardia, Sinus bradycardia, Absent P wave, Axis change, Voltage changes, Electrical alternans, ST depression, ST elevation, Infarction like pattern, T flat/T inversion

e) ECG changes after insulin administration: ECG changes and arrhythmias were not noticed after insulin administration in $68 \%$ of the animals whereas in the remaining $32 \%$ of the animals it took 20 minutes after insulin administration for the arrhythmias like nodal premature contractions, first degree heart block, nodal rhythms and other changes like wide QRS, T inversion and low voltage to revert towards normal [26] [29] [30].

3) ECG studies using Leiurus quinquestriatus venom

The i.v. injection of Leiurus quinquestriatus venom $(1 \mathrm{mg} / \mathrm{kg})$ into rabbits caused an initial moderate bradycardia and immediate inversion of the $\mathrm{T}$ wave in $\mathrm{I}$. This was either accompanied or followed by inversion of $\mathrm{T}$ wave in III, aVL, aVF, $\mathrm{V}_{1}$ and $\mathrm{V}_{5}$. In most animals, the ST segment was depressed in I, II, aVL and $\mathrm{V}_{5}$. A 
notched R wave in I or an RSr' pattern in II was seen at the same time.

The bradycardia progressed with time and became very severe near the time of death, which generally occurred 10 - 50 min following venom injection.

Other electrocardiographic abnormalities included a prominent $\mathrm{Q}$ wave in $\mathrm{I}, \mathrm{aVR}, \mathrm{V}_{1}$ and $\mathrm{V}_{5}$. Some dropped beats and occasional coupled beats. Bigeminy was very prominent during the phases of marked bradycardia.

Tall peaked and slender T wave, S wave $>\mathrm{R}$ wave, prolonged ST interval and wide QRD complexes were frequently observed. When the venom was injected into animals maintained under artificial ventilation, neither the electrocardiographic changes nor the death time were altered [6]-[8].

4) Electrocardiographic studies using A. amoreuxi venom

A. amoreuxi venom in doses lower than $0.5 \mathrm{mg} / \mathrm{kg}$ did not cause any significant ECG changes, while $1 \mathrm{mg} / \mathrm{kg}$ i.v. and higher doses caused definite changes within 30 - 120 minutes. The first effect noticed was a short-lasting bradycardia which changed to slight tachycardia after 2 - 3 min. Some arrhythmias were observed in some animals. The tachycardia was over within 5 - 15 min and was followed by bradycardia which persisted until death of the animal. The ST Segment was elevated in I, aVL and $V_{5}$ but depressed in II, III and $V_{1}, 3$ min after venom injection. Ten minutes after venom injection, the T wave was inverted in I, II, III and aVL and inverted or biphasic in $\mathrm{V}_{1}$. Some extra systoles were seen in I, II, III, and aVL. Thirty minutes after venom injection the T wave was inverted in I, aVL. The ST segment was depressed in I and V5. The inversion of the T wave in I, aVL and V1 was more prominent 60 min after venom injection. Ninety minutes after venom injection there was very marked bradycardia with prolonged P-R intervals. There was no sinus rhythm and 2:1, 3:1 and several rhythms were seen. The ST Segment was elevated in I and V5. This was accompanied in most cases by respiratory arrest leading to death of the animal [6]-[8].

5) Route of administration of venom and ECG changes

When the venom was injected s.c. (rabbits) or i.m. (rabbits), the onset of the ECG effects and death were delayed although the course of the change was the same as the i.v. route. The bradycardia occurred $40-60 \mathrm{~min}$ following the s.c. injection of the venom and somewhat longer following the i.m. route [12]. Postmortem examination of the animals showed large infarcted areas either in the antero-septal or inferior aspects of the heart whether the venom was given s.c. or i.m.

The most striking ECG changes caused by the venom seemed to be myocardial ischemia and anterior wall infarction. The myocardial ischemia was evidenced from depression of the ST segment in II, III and $V_{1}$. The anterior wall infarction was revealed from elevation of the ST segment in I, aVL and $\mathrm{V}_{5}$ and the inversion of the $\mathrm{T}$ wave in $\mathrm{I}, \mathrm{aVL}$ and $\mathrm{V}_{1}$. These changes occurred 30 - 60 minutes following venom injection [6]-[8].

The ECG effects of the scorpion venom are markedly influenced by electrolyte disturbances; the effects are aggravated by hyperkalemia and hypocalcemia [13].

6) Electrolyte changes and ECG in scorpion envenoming Electrolyte

Scorpion venoms appeared to cause some of their ECG abnormalities through electrolyte changes.

We have reported hyperkalemia [21], hyperglycemia [16]-[30] and hypocalcemia [23] in the experimental animals with scorpion envenoming.

a) Hypocalcemia

The prolonged ST segment or $\mathrm{QT}_{\mathrm{C}}$ interval in addition to the $\mathrm{S}$ wave $>\mathrm{R}$ wave is characteristic of Hypocalcemia. The tall and peaked $\mathrm{T}$ waves and the prolonged $\mathrm{QT}_{\mathrm{C}}$ interval were also recorded in all the victims of yellow scorpion stings.

The effects of autonomic stimulation usually mask those of electrolyte changes. It seems likely that vagal stimulation potentiates, while sympathetic stimulation masks the effect of electrolyte changes in the ECG recordings [6]-[8] [23].

b) Hyperkalemia scorpion envenoming

Hyperkalemia could be due to cardiac sarcolemmal defects

There was an increase in serum potassium levels in dogs after venom injection [21]. There is enough evidence to show that these animals also had cardiac sarcolemmal defects indicated by altered $\mathrm{Na}^{+}-\mathrm{K}^{+}$ATPase, $\mathrm{Mg}^{++}$ ATPase and $\mathrm{Ca}^{2+}$ ATPase activities and a reduction in $\mathrm{Na}^{+}-\mathrm{K}^{+}$ATPase activity of erythrocytes [22]. These results indicate that the cell membrane is leaking, and this could be the reason for hyperkalemia.

Hyperkalemia could be seen by the tall, peaked, and slender T waves, and the wide QRS complex characteristic of Hyperkalemia. Hyperkalemia is known to produce atrio-ventricular abnormalities and AV block, Junctional or ventricular rhythms and terminally ventricular fibrillation. Few of the venom treated animals had these 
types of ECG abnormalities. Amoreuxi venom caused a significant fall in serum potassium level 15 min after venom injection, returned to the pre-injection level $5 \mathrm{hr}$ after injection and became significantly higher than the pre-injection level at $7 \mathrm{hr}$ after injection [6]-[8].

\section{Factors That Cause Severity of Intoxication and Death in Envenoming Syndrome}

\subsection{Severity of Intoxication}

The severity of intoxication is related to the scorpion's species (Buthidae family), age, nutritional state, and to seasonal variations and geographical area.

The severity of intoxication is related to the amount and composition of the venom. The in vitro composition may be further influenced by the method used for extracting the venom and the method of purification [36].

1) Enzymes in the scorpion venom

Scorpion venom consists of a mixture of many pharmacologically active proteins. Some proteins are enzymes and others are non-enzymatic in nature [46]. Scorpion venoms beside proteins contain non-protein substances like free amino acids, Histamine, Serotonin, Tryptamine, Glycosaminoglycans, Chondroitin sulphate, Hyaluronic acid, and Free hexosamines

Enzymes are known to have: Gelatinolytic, Thrombin-like, Phospholipase, Hyaluronidase, Proteinase, Peptidase, and Urease activities. Enzymes like Phospholipase $\mathrm{A}_{2}$, acetylcholinesterase, Hyaluronidase, phosphomonoestearase, 5-nucleotidase, gelatinase were reported in various scorpion venoms [48]-[51].

Mesobuthus tamulus concanesis, Pocock venom contained numerous biologically active components. It contains four histamine releasing factors, three neurotoxins and one protease inhibitor [49]. The venom (Buthus tamulus) is a complex water soluble substance containing muco-polysacharides, small amounts of Hyaluronidase and Phospholipase, low molecular weight molecules such as serotonin, histamine, protease inhibitors, histamine releasers and neurotoxins. The neurotoxins have been purified, characterized, and sequenced. These toxins are basic polypeptides with low molecular weight, constituting a single polypeptide reticulated by four disulfide bridges. Scorpion neurotoxins influence mainly excitable membranes releasing neurotransmitters from neurons, ganglia, and nerve endings [50].

2) Scorpion Toxins: Pulmonary oedema producing toxin

Mesobuthus tamulus concanesis, Pocock venom contains a potent cardiopulmonary toxin, which could account for the manifestation of pulmonary oedema seen after the scorpion envenomation. By Sephadox gel filtration and cation exchange chromatography, a high molecular weight toxin (T3) exhibiting the toxicity on cardio pulmonary parameters was isolated from Mesobuthus tamulus concanesis, Pocock venom was named as "Pulmonary oedema producing toxin". Another lethal fraction was also isolated (T2), which is a low molecular weight toxin and exhibited neurotoxicity [50].

3) Neurotoxins

Scorpion venom's neurotoxins affect the gating mechanisms of excitable membrane.

Long toxins (60 - 70 amino acids) interfere with sodium channels, causing cell destruction by the influx of $\mathrm{Na}^{+}$and $\mathrm{Ca}^{2+}$ ions into the cell.

Short toxins (31 - 37 amino acids) modify the potassium channel's function and may specially block $\mathrm{Ca}^{2+} \mathrm{ac}^{-}$ tivated $\mathrm{L}^{+}$channels. These effects on vascular endothelial cells may exert different reactions on different organs [51].

4) Natriuretic peptide

Scorpion envenomation is considered public health problem in North African countries. The North African scorpion toxin from Androctonus australis garzonii increases the Atrial Natriuretic peptide (ANP) in rat through stimulation of sympathetic cardiac nerves and sodium channels activation [48] [51].

5) Tityustoxin acts by activating the sodium channels

Experimental studies have established that "Tityustoxin", a highly purified fraction of the venom of $T$. serrulatus, acts by activating the sodium channels, causing persistent membrane depolarization in the excitable cells of the organism. This leads to a massive release of neurotransmitters by the sympathetic and Para-sympathetic nerve endings and also by the adrenal medulla, which in turn causes most of the signs and symptoms in human envenoming [52].

6) $\mathrm{Na}^{+}$channel blocking peptides

Sodium channels are the most common target for scorpion neuro-peptides, and these neuro-toxins are organized into three groups [53]: the alpha toxins, the beta toxins and the gamma toxins. 
The alpha toxins are commonly found in the "Old World" scorpion species (Androctonus), and function by preventing the closure of the voltage gated sodium channels in excitable cells. This results in repetitive firing [53].

The beta toxins are commonly found in the "New World" scorpion species (Centruroides and Tityus) and block/ delay the opening of the channel, inhibiting the nerve conduction [54].

Toxin $y$, found in the Brazilian scorpion Tityus serrulatus, accomplishes both tasks, as it blocks the opening and closing of sodium channels [55].

Posani et al., working with Mexican scorpions reported the action of the venom from the genus Centruroides on peripheral nervous system and muscle preparations. A drastic decrease on the ionic permeability properties of $\mathrm{Na}$ and $\mathrm{K}$ channels of the node of Ranvier, caused by application of scorpion venoms was observed by many investigators. Most of the work was devoted to the Na-channel blocking peptides, major components of scorpion venoms, assumed to be the principal cause of human envenomation due to stings by these arachnids [56].

7) $\mathrm{K}^{+}$channel blocking peptides from the venom of scorpions

$\mathrm{K}^{+}$channel blocking toxins belong to a low molecular weight peptide family, with exquisite properties towards $\mathrm{K}^{+}$channels, opening the possibility to dissect the intricate molecular mechanisms of action of one of the most diverse receptors present in excitable membranes-the $\mathrm{K}^{+}$channels [56].

Sodium channel specific toxins are voltage gated. But potassium channel toxins have both voltage-gated and ligand-gated toxins. Potassium channel toxins have two sub-units. Alpha sub-unit is responsible for ion conduction across the membrane. Beta sub-unit is responsible for modulating the properties of the channel [57].

Toxins affecting neuronal $\mathrm{K}^{+}$channels have attracted considerable interest. They include noxiustoxin from the Mexican scorpion Naxius Centruroides and Charybdotoxin from the Old World scorpion Leiurus quinquestiatus. These toxins are highly potent blockers of $\mathrm{K}^{+}$currents in neurons, but they differ in selectivity for subtypes of $\mathrm{K}^{+}$channels [53].

Potassium channels are found in nearly every excitable tissue, and play important roles in neurotransmitter release. Potassium channel specific toxins are thought to completely block potassium channels [38] [58]. Noxiustoxin (NTX) was the first peptide purified and sequenced was shown to affect the voltage-dependent K channel of axons.

8) Low molecular weight peptides specific for $\mathrm{K}^{+}$channels

Charybdotoxin (Chtx), a basic polypeptide from the Asian scorpion Leiurus quinquestriatus, originally described as a blocker of the Ca-dependent $\mathrm{K}$ channel.

Leiurotoxin (LeTx), a low conductance, apamin-sensitive $\mathrm{K}^{+}$channel blocker from the same venom.

Iberotoxin (IbTx), a high conductance Ca activated K channel from the venom of the scorpion Buthus tamulus [56].

Serrulatoxins, from the venom of the Brazilian scorpion Tityus serrulatus three new low molecular mass peptides (Serrulatoxins) were isolated and shown to affect the Ca dependent $\mathrm{K}$ channels of skeletal muscle.

NTX -like peptides: two new NTX_like peptides were purified from the venom of the scorpion Centruroides limpidus limpidus.

Noxiustoxin (NTX) was the first peptide purified and sequenced was shown to affect the voltage-dependent K channel of axons.

Excitability of neurons-repetitive action potentials

The toxins that affect the activation or inactivation of $\mathrm{Na}^{+}$channels can cause an increase in the excitability of neurons, leading to repetitive action potentials. This can lead to an increase in transmitter release. The toxins can produce a sustained depolarization of nerve membranes and nerve terminals. This can block action potential conduction in axons, and cause an uncontrolled release of transmitter from nerve endings.

Tityus zulianus (Venezuelan scorpion) has beta toxin that works on skeletal muscle sodium channels [52].

\subsection{Integrity of the Stinger}

In addition to the quantity and composition of the venom, the integrity of the stinger of the offending scorpion is important.

\subsection{Yield of the Venom from the Scorpion during "Milking"}

There are considerable differences in the yield of the venom among different scorpions of the same species dur- 
ing “milking”. Values of $0.65 \mathrm{mg}$ and 0.9 - $1.5 \mathrm{mg}$ dry venom were obtained from Leiurus quinquestiatus and its sub-species $B$. quinquestiatus, respectively. Values of $0.8-1.2 \mathrm{mg}$ dry venom in adults, and up to $2.00 \mathrm{mg}$ in pregnant Leiurus quinquestiatus were obtained by Ismail [8].

\subsection{Size and Age of the Victim Is of Utmost Important}

The size and age of the victim is of utmost important. Thus, children and babies are more prone to severe intoxication (dose dependent). Other factors are the site of injection and, possibly differential vulnerability to the venom [36].

\subsection{Toxicity of Scorpion Venom in Mammals Is Influenced by the Age and Species}

Deaths due to Indian red scorpion (Mesobuthus tamulus concanesis, Pocock) stings occur in both adults and children, but the mortality is greater in children. Besides the age, species of animals is also an important factor for the sensitivity of Mesobuthus tamulus concanesis, Pocock venom. The toxic effects of the venom are dissimilar at different doses, e.g. a low dose depleted the liver or ventricular muscle glycogen to a greater extent than did a higher dose in rabbits [59].

\section{6. $\mathrm{LD}_{50}$ Value}

The $\mathrm{LD}_{50}$ value for the scorpion (Mesobuthus tamulus concanesis, Pocock) venom in young rats was $170 \%$ of the adult rats. The $\mathrm{LD}_{50}$ value for guinea-pig and mice were $88 \%$ and $550 \%$ of adult rat, respectively. The acute s.c. $\mathrm{LD}_{50}$ value for Leiurus quinquestiatus of the venom for male mice was $0.25 \mathrm{mg} / \mathrm{kg}$ [6].

The $\mathrm{LD}_{50}$ of a given scorpion venom is often reported differently by different investigators depending on the geographical location, method of obtaining the venom, species of the mice used, route of venom injection. An example is the venom from the scorpion Leiurus quinquestiatus which has the followingLD $\mathrm{D}_{50}$ values $(\mathrm{mg} / \mathrm{kg}$ ) reported by different workers as shown below [7].

$0.80 \mathrm{mg} / \mathrm{kg}=$ Tetsch and Wolf, 1937,

$5.09 \mathrm{mg} / \mathrm{kg}=$ Shulov et al., 1957,

$6.50 \mathrm{mg} / \mathrm{kg}=$ Adam and Weiss, 1959,

$0.33 \mathrm{mg} / \mathrm{kg}=$ Bucherl, 1971,

$0.25 \mathrm{mg} / \mathrm{kg}=$ Zlotkin et al., 1978,

$0.26 \mathrm{mg} / \mathrm{kg}=$ Delori et al., 1981,

$0.23 \mathrm{mg} / \mathrm{kg}=$ Ismail et al., 1992 .

\subsection{Young Rats Required Greater Concentration of Scorpion Venom than the Adult Rats}

Young rats required greater concentration of scorpion (Mesobuthus tamulus concanesis, Pocock) venom than the adult rats; thus enhanced sensitivity to venom in young animals may not be likely to explain the greater mortality. Tiwari \& Deshpande observed dose-dependent fatalities on a weight basis; thus it is possible that the concentration of venom (per kg body weight) per sting will be greater in the young than in the adults, perhaps accounting for the greater casualties in children, even though they may be less sensitive to venom [59].

\subsection{Failure of the Autonomic Nervous System}

Scorpion envenomation is known to produce severe autonomic storm. It is known that the blood-brain barrier is not fully developed in new born infants and young children and as a consequence there is increased permeability of certain substances into the brain, particularly the general anesthetics, sedatives, narcotic analgesics, and un-conjugated bilirubin. The autonomic system is still in the developing stage in children; therefore, failure of the autonomic nervous system is also a possibility and scorpion venom could cross the blood-brain barrier of infants and children [6].

\subsection{Site of Scorpion Sting}

Scorpions usually sting at the web spaces between the fingers, tips of fingers, toes and any other anatomical region of the body of the victim. Web spaces between the fingers, tips of fingers and toes are highly vascular. 
Scorpion stings may result in injection of its venom either sub-cutaneously, intramuscularly or intravenously.

\subsection{Subcutaneous (S.c.) Route Is Known to Evoke Greater Cutaneous Sensory Stimulation than the Intra-Muscular (I.M.) Route}

1) Subcutaneous (s.c.) route

2) Intra-muscular (i.m.) route

The $\mathrm{LD}_{50}$ values reported for the intramuscular route for scorpion (Mesobuthus tamulus concanesis, Pocock) venom [46] were higher than the values of s.c. route reported by Tiwari \& Deshpande [59]. The subcutaneous route is known to evoke greater cutaneous sensory stimulation than the i.m. route, and this might be responsible for such variation.

3) Intravenous injection of the venom is extremely lethal

Intravenous injection of the venom is extremely lethal in rats (Charles Foster) and doses as low as 120 micrograms $/ \mathrm{kg}$ killed all the animals, but in Sprague-Dawley rats the $\mathrm{LD}_{100}$ value (dose) is 2.5 - 16.5 times greater than the values observed by Tiwari \& Deshpande [59]. Radha Krishna Murthy et al. used intravenous dose of scorpion (Mesobuthus tamulus concanesis, Pocock) venom (2 - $4 \mathrm{mg} / \mathrm{kg}$ body weight) for their experimental dogs and rabbits [16]-[27].

4) Greater fatalities in children

The greater fatalities in children following scorpion envenomation might be due to various factors, namely greater sensitivity to venom or relatively greater quantity of venom on weight basis being injected into the victim, or to the venom-induced alterations in the body systems (reaction to pain, autonomic storm, cardio-respiratory and nervous system changes), acting either singly or in combination [59].

\section{Functions and Effects of Renin-Angiotensin System}

\subsection{Angiotensin May Initiate Reflex Vagal Activity-Sinus Bradycardia or Sinus Arrest}

By increasing systemic blood pressure and baroreceptor discharge, angiotensin may initiate reflex vagal activity sufficient to slow the heart rate. This could explain the sinus bradycardia or sinus arrest coinciding with the peak of blood pressure elevation in animals after venom injection [1]-[8] [20]-[26] [34]-[60].

\subsection{Peripheral Circulatory Failure}

Angiotensin appears to constrict post-capillary vessels and increase filtration pressure in the capillaries, a significant diminution in the blood volume and increase the extra vascular fluid. This could be the reason for the peripheral circulatory failure in the scorpion sting victims [1]-[8] [20]-[26] [34]-[60].

\subsection{Mechanism of Changes in the Mean Arterial Blood Pressure Due to Scorpion Venom}

Initial Transient Hypertension in Scorpion Envenoming

Initial transient hypertension is observed in our poisoned animals (Figure 1). Mesobuthus tamulus Concanesis, Pocock venom raised plasma angiotensin levels in the experimental dogs (225\%) and rabbits (1178\%) [19]. During the Phase of initial transient hypertension the mean arterial blood pressure increased to more than 260 $\mathrm{mm}$ Hg. The time duration of this phase was very short and thereafter the blood pressure dropped slowly and showed a Phase of hypotension.

\subsection{Hypotension in Scorpion Envenoming}

Hypotension (after the initial transient hypertension) following scorpion envenoming is a common observation in the experimental animals and scorpion sting victims.

Gueron et al. reported catecholamine metabolites excretion in 12 scorpion sting victims. Abnormal serum level and increased catecholamine metabolite urine excretion was also reported. Elevated circulating levels of catecholamines and rennin angiotensin had been observed in clinical and experimental envenomation [1]-[5] [19] [33]-[60].

The changes in the blood pressure could be explained as due to autonomic storm resulting from injection of venom and subsequently releasing great quantities of catecholamines. 


\subsection{Mechanism of the Rise in Blood Pressure Due to Nor-Epinephrine and Epinephrine}

1) A direct myocardial stimulation that increases the strength of ventricular contraction (Positive inotropic action);

2) An increased heart rate (Positive chrono-tropic action);

3) Vasoconstriction in many vascular beds-especially in the pre-capillary resistance vessels of skin, mucosa, and kidney-along with marked constriction of the veins;

4) Nor epinephrine and epinephrine are direct agonists on effector cells, and their actions differ mainly in the

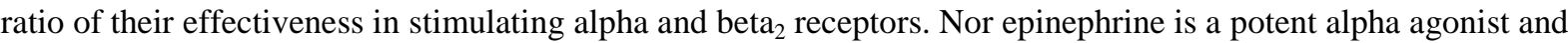
has relatively little action on beta ${ }_{2}$ receptors [33]-[60].

\subsection{Renin-Angiotensin System}

A major role in the short term regulation of arterial blood pressure.

The renin-angiotensin system plays a major role in the short term regulation of arterial blood pressure. Modest increases in plasma concentrations of angiotensin II acutely raise blood pressure. Angiotensin II is 40 times more potent than nor-epinephrine. Systemic blood pressure begins to rise within seconds, peaks rapidly. This rapid pressor response to angiotensin II is due to a swift increase in total peripheral resistance [60].

\subsection{Angiotensin II Increases Total Peripheral Resistance}

Angiotensin II increases total peripheral resistance via direct and indirect effects on blood vessels. Angiotensin II constricts pre-capillary arterioles and, to a lesser extent, post-capillary venules. Direct vasoconstriction is strongest in the kidneys and somewhat less in the splanchanic vascular bed. High circulating concentrations of angiotensin II may decrease cerebral and coronary blood flow [60].

\subsection{Angiotensin II-Enhancement of Peripheral Noradrenergic Neurotransmission}

In humans, angiotensin II potentiates sympathetic nervous system-induced coronary vasoconstriction.

Angiotensin II facilitates peripheral noradrenergic neurotransmission by augmenting nor-epinephrine release from sympathetic nerve terminals, by inhibiting the reuptake of nor-epinephrine into nerve terminals, and by enhancing the vascular response to nor-epinephrine. High concentrations of angiotensin II stimulate ganglion cells directly. In humans, angiotensin II potentiates sympathetic nervous system-induced coronary vasoconstriction [60].

\subsection{Angiotensin II-Effects on the Central Nervous System}

Circulating angiotensin II causes an increase in arterial blood pressure. This response mediated by increased sympathetic outflow-reflects effects of angiotensin II on circumventricular nuclei that are not protected by a blood-brain barrier. Circulating angiotensin II also attenuates Baroreceptor-mediated reductions in sympathetic discharge, thereby increasing arterial pressure. The CNS is affected both by blood-borne angiotensin II and by angiotensin II formed within the brain. The brain contains all components of a renin-angiotensin system. Moreover, there is angiotensin like immunoreactivity at many sites within the CNS, suggesting that angiotensin II serves as a neurotransmitter or modulator [60].

\subsection{Angiotensin II-Release of Catecholamines from the Adrenal Medulla}

Angiotensin II stimulates the release of catecholamines from the adrenal medulla by depolarizing chromaffin cells [1]-[10] [12]-[15] [32]-[60].

\subsection{Angiotensin II-Synthesis and Release of Aldosterone Is Enhanced under Conditions of Hyponitremia or Hyperkalemia}

Angiotensin II stimulates the zona glomerulosa of the adrenal cortex to increase the synthesis and secretion of aldosterone, and Angiotensin II exerts trophic and permissive effects that augment responses to other stimuli. The stimulant effect of Angiotensin II on aldosterone synthesis and release is enhanced under conditions of hypo-nitremia or hyperkalemia. We have demonstrated hyperkalemia in experimental scorpion envenoming [21]. 


\section{Autonomic Storm, Release of Counter Regulatory Hormones and Suppressed Insulin in Severe Scorpion Envenoming}

Severe scorpion envenoming causes an increase in the circulating levels of blood sugar, insulin, glucagon, and Cortisol. Mesobuthus tamulus Concanesis, Pocock venom injection (s.c.) in the dogs caused hypo-insulin secretion and elevated insulin levels at $30 \mathrm{~min}$ and at $60 \mathrm{~min}$ respectively after venom injection. Insulin and blood glucose were high at after 60 and 120 min after venom injection [17] [21]-[24] [26] [29].

1) Glycogenolysis-hyperglycemia

2) Increase protein breakdown products

3) Lipolysis sudden increase in Free Fatty Acids

In the presence of increased circulating levels of all these catabolic counter-regulatory hormones, the effects of these hormonal actions are synergistic and sustained hepatic glucose production is observed. The simultaneous elaboration of the counter-regulatory hormones is partly responsible for the pathogenesis of a variety of clinical and biochemical manifestations following scorpion envenoming. This could be the reason for glycogenolysis in the atria, ventricles, and liver; and skeletal muscles [16] [17] [21]-[24] [26] [27] [29]; hyperglycemia [16] [17] [21]-[24] [26] [27] [29]; lipolysis and elevated free fatty acid levels [16]-[18] [21]-[24] [26] [27] [29], increased protein breakdown products under the catabolic influence of the counter-regulatory hormones, and a simultaneous suppressed insulin secretion or insulin resistance.

4) Hyperinsulinemia-insulin resistance

Hyperinsulinemia observed in our studies could be equated with insulin resistance. Insulin resistance could be caused by a change in the receptor membrane, a change in hormone-receptor binding characteristics, or a change in the post receptor events.

5) Catabolic state with low Insulin/glucagon (I/G) ratio

When $\mathrm{I} / \mathrm{G}$ ratio are low, a catabolic state is produced in which nutrients are mobilized. Scorpion envenoming causes a low I/G ratio [17].

6) Highly abnormal Renin and aldosterone levels

Catecholamine-Renin-angiotensin and aldosterone levels, glucagon, Cortisol, abnormal insulin secretions are observed in scorpion envenoming. The metabolic actions of all these hormones cause suppressed insulin secretion, or hyper-insulinemia/insulin resistance resulting in hyperglycemia and sudden increase in Free Fatty Acids.

\section{Toxicity Due to Sudden Increase in Free Fatty Acid Levels in Scorpion Envenoming}

\subsection{Causes of Sudden Increase in Free Fatty Acid Levels}

1) Role of Glucagon

Glucagon is a powerful lipolytic agent, acting via cAMP to phosphorylate a lipase in adipose tissue which releases FFA and glycerol in to circulation [17][57]-[60] [61]-[63].

2) Role of Adrenalin and Nor-adrenalin

Adrenalin and noradrenalin activate specific lipase in adipose tissue and muscle which breaks down triglycerides to FFA and glycerol. This action is antagonized by insulin [63].

3) Lipolytic action of adrenaline is brief and the action of noradrenalin is prolonged

The catecholamines make available for active tissue more oxidizable structures, such as FFA, glycerol, and ketone bodies, and at the same time depress the oxidation of glucose. The lipolytic action of adrenaline is brief and the action of noradrenalin is prolonged. The catecholamines promote lipolysis in adipose tissue and proteolysis in muscle [63].

\subsection{Effect of Increased Free Fatty Acids on the Heart}

1) Increased oxygen consumption

The use of increased amounts of Free Fatty Acids results in increased oxygen consumption. This could aggravate the ischemic injury to myocardium, predisposing to arrhythmias and heart failure.

2) Free Fatty Acids increase the susceptibility of the ventricles to the disorganized electrical behavior

The elevated free fatty acids also increase the susceptibility of the ventricles to the disorganized electrical be- 
havior and produce ectopic beats in the vulnerable period of cardiac cycle [64].

3) Free Fatty Acids inhibit $\mathrm{Na}^{+}-\mathrm{K}^{+}$ATPase activity

High levels of free fatty acids produce inhibition of $\mathrm{Na}^{+}-\mathrm{K}^{+}$ATPase activity [31] and produce cardiac sarcolemmal defects. Alterations in cardiac sarcolemmal $\mathrm{Na}^{+}-\mathrm{K}^{+}$ATPase, $\mathrm{Mg}^{++}$ATPase and $\mathrm{Ca}^{2+}$ ATPase activities indicate cardiac sarcolemmal defects [22].

4) Free Fatty Acids increase the tendency to intravascular thrombus

The increased Free Fatty Acids, by altering the functions of platelets, may increase the tendency to intravascular thrombus and result in disseminated intravascular coagulation [23] [65].

5) Free Fatty Acids increase myocardial oxygen consumption

Scorpion stings can be accompanied by enzymatic and electrocardiographic evidence of various types of myocardial cellular damage. Echocardiographic and radionuclide studies in human victims have shown depressed L.V. systolic function in patients tested within a few hours of scorpion sting [1]-[8] [12]-[15] [34] [35] [40]-[43].

6) $\mathrm{O}_{2}$ demand/supply ratio

Myocardial ischemia immediately following administration of scorpion venom is related to increased myocardial oxygen consumption associated with catecholamine release coupled with a decrease in oxygen supply due to transient reduction of coronary blood flow. Thus, immediately post-envenomation, the myocardial $\mathrm{O}_{2}$ demand/ supply ratio would be adversely affected, resulting in ischemia [1]-[8] [10] [12]-[18] [23]-[26] [64] [66].

7) The metabolism of normal myocardium and the role of insulin

Under normal conditions, the myocardium derives its energy from the aerobic metabolism of substrates extracted from the plasma. The most important fuels are Free Fatty Acids (FFA), glucose, triglycerides, amino acids, Pyruvate and lactate. At rest, the myocardial extraction of many of these substrates is generally related to their arterial concentrations, but the relative uptake of each compound may be modified by hormones and utilization of other substrates. Thus, glucose transport into the myocardial cell depends on insulin, and increases of plasma insulin concentration enhance the extraction of glucose by the heart [64] [66]-[69].

8) The metabolism of injured myocardium and the role of insulin

High plasma FFA concentrations inhibit glucose uptake by the heart. We have consistently demonstrated a sudden increase in FFA levels (200\% to 300\%) in the experimental scorpion envenoming [17] [18] [23]-[26] [29] [70] [71].

9) The metabolism of injured myocardium

High plasma FFA concentration increases myocardial oxygen consumption without augmenting mechanical performance. High plasma FFA levels in patients with myocardial infarction are associated with increased incidence of arrhythmias, decrease heart contractility, and increase the extent of myocardial damage [17]-[19] [23]-[29] [69]-[71].

10) Effects of acute ischemia on myocardial metabolism

The immediate metabolic changes in the myocardium during acute ischemia are largely determined by the rates of glycolysis and glycogenolysis and to a lesser extent, of fatty acid availability in relation to the demand for phosphorylation.

Glycolysis increases with mild hypoxia, and in areas of profound hypoxia, decreased glycogenolysis occurs. Hydrolysis of stored triglycerides results from the activation of myocardial lipase with increases in FFA. Greater glycogenolysis was observed in atria and ventricular tissue in response to a smaller dose of scorpion venom compared to lower rate of glycogenolysis with a higher dose of scorpion venom in our experimental animals.

The ischemic myocardium extracts proportionately more FFA than at lower plasma concentrations. The ischemic myocardium is presented, therefore, with a considerable excess of FFA relative to glucose and, in a severely ischemic zone, the available oxygen may be insufficient for oxidation [17]-[19] [23]-[29] [63]-[71].

11) Myocardial vulnerability

While myocardial vulnerability may finally be determined by critical extra and intra cellular gradients in the availability of $\mathrm{Ca}^{2+}, \mathrm{Mg}^{++}$and $\mathrm{K}^{+}$, the intracellular concentrations of these ions, are in turn, dependent on factors which influence their transference across the cell and mitochondrial membranes. A decreased availability of $\mathrm{Ca}^{2+}$ can interfere with Actin-Myosin coupling so that contractility is impaired.

An excess of $\mathrm{K}^{+}$could alter the action potential so that self perpetuating re-entry currents are established [69].

Low concentrations of myocardial $\mathrm{Mg}^{++}$are associated with arrhythmias and sudden death [64] [66] [69]. 
12) Accumulation of excess intra cellular FFA: Detergent effect on cell membranes

Accumulation of excess intra cellular FFA could have a detergent effect on cell membranes [31] Elevated concentrations of plasma FFA have been associated with an increased prevalence of serious ventricular arrhythmias and death in man and in dogs. High plasma FFA levels increased ST elevation in the ECG and plasma CK enzyme levels, suggesting that they may lead to more extensive damage [64] [66] [69].

13) Excess un-oxidized FFA probably become toxic to the myocardium

Excess un-oxidized FFA probably becomes toxic to the myocardium only when there is acute ischemia.

14) Interfere with tropomyosin-troponin activation of Actin-Myosin coupling

The biochemical consequences which result from the intra cellular accumulation of excess un-oxidized FFA for $\mathrm{Ca}^{2+}$ will occur simultaneously with protein binding, possibly making less ionic calcium available for passage into the sarcotubular reticulum. This might interfere with tropomyosin-troponin activation of Actin-Myosin coupling [64].

15) Intra cellular depletion of $\mathrm{Mg}^{++}$would be exaggerated

Magnesium-fatty acid complexes could result and any intra cellular depletion of $\mathrm{Mg}^{++}$would be exaggerated. This might lead to uncoupling of oxidative phosphorylation, possibly by interfering with a Magnesium-dependent ATPase system $\left(\mathrm{Mg}^{++}\right.$ATPase) [22] [69]. Cardiac sarcolemmal $\mathrm{Na}^{+}-\mathrm{K}^{+}$ATPase and erythrocyte $\mathrm{Na}^{+}-\mathrm{K}^{+}$ ATPase are Magnesium-dependent ATPase systems [22]. We have demonstrated alterations in these enzyme activities after scorpion envenoming.

16) Excess un-oxidized fatty acids or metabolites could alter the stabilization of lysosomal membranes

Fatty acids may be transported intra-cellularly in the un-esterfied form and have specific affinities for certain subcellular structures. Excess un-oxidized fatty acids or metabolites could alter the stabilization of lysosomal membranes and mitochondrial integrity, and together they can have a synergistic effect [29] [64] [69] [71].

17) Elevated FFA levels and incidence of different types of arrhythmias, conduction defects, ischemia, and infarction like patterns in ECG

Elevated FFA levels [17]-[26] with incidence of different types of arrhythmias, conduction defects, ischemia, and infarction-like patterns in ECG have been shown in our experimental animals with scorpion envenoming and in scorpion sting victims [1]-[10] [12]-[35] [37]-[44] [65] [70] [71].

18) Increased FFA oxidation can inhibit glycogen synthatase activity directly

Increased FFA oxidation can inhibit glycogen synthatase activity directly by causing a dissociation of its subunits [63].

19) Elevated rate of FFA oxidation can reproduce all major intracellular abnormalities

An elevated rate of FFA oxidation can reproduce all major intracellular abnormalities (decreased glucose transport, decreased glycogen synthatase, decreased Pyruvate Dehydrogenase and could account for the defects in glucose oxidation and storage. Physiological elevations in plasma FFA concentrations cause a stimulation of FFA oxidation, which in turn, inhibits glucose oxidation and storage [63] [66] [69].

\section{Insulin Administration}

\subsection{Insulin Administration in Experimental Envenoming Resulted in a Reduction in FFA Levels, Disappearance of Different Arrhythmias, Conduction Defects, Ischemia, and Infarction-Like ECG Patterns with Normal Sinus Rhythm}

We have reported a reduction in FFA levels, disappearance of different arrhythmias, conduction defects, ischemia, and infarction-like ECG patterns with normal sinus rhythm after insulin administration in experimental envenoming [24] [26] and in scorpion sting victims [11] [27] [29] [30] [71].

\subsection{Insulin Counteracts All the Deleterious Effects of FFA}

1) Inhibiting the catecholamine-induced lipolysis in the adipose tissue, thus reducing the plasma FFA level;

2) Facilitating the glucose transport to the myocardium and glucose metabolism through different pathways; and

3) Increasing the intracellular potassium concentrations;

Insulin administration reversed metabolic changes and other abnormalities due to envenoming. Insulin stimulates activation of glycogen synthetase system. This could be the reason for an increase in glycogen content of 
cardiac, skeletal muscles and liver of the insulin, alpha blocker + sodium bicarbonate treated animals after envenomation. Moreover, glycogen availability may be an important independent determinant of cardiac function. Elevated glycogen in heart partially protects against mechanical deterioration in anoxia [72].

Insulin stimulates glycogen synthesis [63]. Thus insulin counter-acts the effects of catecholamines favoring glucose uptake and inhibition of glucose release from liver. This could be the reason for an increased glycogen content of atria, ventricle, liver and skeletal muscle after insulin administration.

Insulin administration suppresses the release of FFA from adipose tissue and this effect is immediate and even faster than the effect on plasma glucose levels [35] [63].

Insulin stimulates lipogenesis [63]. This could be the reason for the sudden reduction of free fatty acid levels and increased triglyceride levels in the venom poisoned animals after administration of insulin. Moreover, infusion of glucose in these animals along with insulin, will suppress fat mobilization by favoring re-esterification.

In our hands, administration of insulin (present study) or insulin + alpha blocker (Tolazodine) + Sodium bicarbonate [39] successfully reversed the metabolic as well as ECG changes.

Insulin + alpha blocker (Tolazoline) + Sodium bicarbonate [24] produced little more glycogenesis and lipogenesis than insulin. However, alpha blockers are known to stimulate the gastric acid secretion and this in turn could aggravate the existing sub-clinical pancreatitis into fully blown up fulminating acute pancreatitis in scorpion sting victims.

Conclusion: The initial transient hypertension followed by hypotension, cardiovascular manifestations, metabolic disturbances, electrocardiographic changes, and adult respiratory distress syndrome and many other clinical manifestations produced by scorpion venom toxicity could be due to

1) Action of catecholamines causing increased myocardial oxygen consumption due to positive and chronotropic effects, coronary vasoconstriction, peripheral vasoconstriction and increased after load, Lipolysis resulting in increased FFA;

2) Action of angiotensin II resulting in coronary and peripheral vasoconstriction, potentiation of catecholamine mediated effects;

3) Insulin deficiency;

4) Increased FFA resulting increased myocardial oxygen consumption; and

5) Arrhythmogenic effect of catecholamines, angiotensin II and free fatty acids.

Insulin administration resulted in glycogenesis, lipogenesis, stopped arrhythmias and reversed the ECG changes to sinus rhythm.

\subsection{Administration of Insulin}

Administration of insulin under these circumstances should counter-act the metabolic effects of catecholamines, stimulate lipogenesis, glycogenesis, reverse the metabolic and electrocardiographic changes in acute myocarditis induced by Indian red scorpion (Buthus tamulus) venom in the experimental dogs.

\subsection{The Dose of Insulin in Scorpion Sting Victims}

The dose of insulin is 0.3 Units of regular insulin per gram of glucose, and glucose $0.1 \mathrm{~g} \cdot \mathrm{Kg}^{-1} \mathrm{per}$ hour. Blood glucose, serum electrolytes, electrocardiogram, and arterial blood gases should be investigated on admission. In addition to regular clinical observations, estimations of blood glucose should be carried out two hourly and of serum electrolytes 12-hourly. Glucose levels should be maintained between 130 and $180 \mathrm{mg} \cdot \mathrm{dl}^{-1}$ of blood.

Insulin administration produced a reduction in FFA, an increase in triglyceride levels and increased tissue glycogen content in cardiac and skeletal muscle and that of liver.

\subsection{Effect of Insulin Administration on "Hypertension"}

The arterial blood pressure came back to normal pressure after insulin administration in the experimental animals and scorpion sting victims.

\subsection{Effect of Insulin Administration on "Hypotension"}

The arterial blood pressure came back to normal pressure after insulin administration in the experimental animals and scorpion sting victims. 


\subsection{Following Drugs Are either Not Useful or Contra-Indicated in Scorpion Envenoming Syndrome}

1) Cardiac glycosides: The cardiac glycosides are not effective in pulmonary oedema in the presence of sinus tachycardia and normal cardiac size. The cardiac glycosides are known to act by inhibiting $\mathrm{Na}^{+}-\mathrm{K}^{+}$ATPase activity. The scorpion venom produces cardiac sarcolemmal defects displayed as inhibition of $\mathrm{Na}^{+}-\mathrm{K}^{+} \mathrm{ATPase}^{-}$activity.

2) Atropine: Atropine should not be given routinely. This has been the common practice because of heavy perspiration and increased salivation.

Atropine may intensify the tachycardia and sympathetic effects due to the venom after blocking the cholinergic effects.

Atropine potentiates hypertensive effect.

Moreover, atropine is a parasympatholytic drug and inhibits insulin secretion from endocrine pancreas.

Increase in duration as well as severity of clinical signs, including myocardial injury were observed in scorpion sting victims treated with atropine compared to scorpion sting victims who did not receive atropine.

Atropine increases the severity of pulmonary oedema induced by scorpion toxin.

3) Diuretics: Diuretics are contraindicated, owing to their dehydrating effect, alteration in blood viscosity and stimulation of rennin-angiotensin secretion.

4) Corticosteroids: Glucocorticoids are contraindicated because they are catabolic hormones and anti-insulin in action. They stimulate the rennin-angiotensin system. Moreover, glucocorticoids act like, "asbestos suit against fire but they themselves do not extinguish the fire”, and in the absence of specific drugs, glucocorticoids are likely to spread the inflammation. Besides, glucocorticoids are contra-indicated in non-cardiogenic pulmonary oedema.

5) Angiotensin Converting Enzyme (ACE) inhibitors: Captopril is an angiotensin-converting enzyme inhibitor that inhibits the conversion of the relatively inactive angiotensin I to the active angiotensin II. It also inhibits the degradation of bradykinin and potentiates its hypotensive action. It is used in the management of hypertension, congestive heart failure and hypertensive emergencies. Captopril and other angiotensin-converting enzyme inhibitors are inferior to other vasodilators in the treatment of heart failure. Ismail, however, cautioned against the use of captopril since the drug inhibits kininase enzyme and thus would lead to the accumulation of bradykinin, the neuro humoral agent, incriminated experimentally for the pulmonary oedema of the scorpion envenoming syndrome.

6) Adrenaline (with local xylocaine injection): Scorpion venom is known to an autonomic storm releasing massive quantities of catecholamine hence adrenaline (with local xylocaine injection) is contraindicated.

\subsection{Treatment}

Scorpion envenoming causes an autonomic storm, release of counter-regulatory hormones along with either suppressed insulin secretion or hyper-insulinemia and a sudden increase in free fatty acid levels. Insulin counteracts all the deleterious effects of FFA by Inhibiting the catecholamine-induced lipolysis in the adipose tissue, thus reducing the plasma FFA level, facilitating the glucose transport to the myocardium and glucose metabolism through different pathways; and increasing the intracellular potassium concentrations.

We consider that insulin has a primary metabolic role in preventing and reversing the cardiovascular, haemodynamic, and neurological manifestations and pulmonary oedema induced by scorpion envenoming syndrome either in the experimental animals or in scorpion sting victims. Administration of insulin-glucose infusion to scorpion sting victims appears to be the physiological basis for the control of the metabolic response when that has become a determinant to survival.

\section{Conclusions}

Scorpion envenoming syndrome is due to autonomic storm releasing massive quantities of catecholamines, angiotensin II, glucagon, Cortisol and either suppressed insulin secretion, or hyper-insulinemia/insulin resistance (failure of the actions of internally secreted insulin). The metabolic actions of all these hormones cause suppressed insulin secretion, or hyper-insulinemia/insulin resistance resulting in hyperglycemia and sudden increase in Free Fatty Acids. Severe scorpion envenoming syndrome is a syndrome of fuel-energy deficits and an inability of the vital organs to utilize the existing metabolic substrates. This ultimately may result in Multi-System 
Organ Failure (MSOF) and death.

Sudden increase in Free Fatty Acids cause sarcolemmal defects reflected in alterations in cardiac sarcolemmal ATPase activities of $\mathrm{Na}^{+}-\mathrm{K}^{+}$ATPase, $\mathrm{Mg}^{++}$ATPase and $\mathrm{Ca}^{2+}$ ATPase activities. These sarcolemmal defects may be responsible for the subsequent pathological conditions. Insulin-glucose infusion resulted in glycogenesis, lipogenesis, stopped arrhythmias and reversed the ECG changes to sinus rhythm in the scorpion envenomed animals and in the scorpion sting victims. Insulin administration following scorpion envenoming reversed the ECG and metabolic changes in the experimental animals as well as in scorpion sting victims. The administration of insulin-glucose infusion should be recommended as the first choice therapy given as soon as possible, immediately after hospitalization in intensive care units to envenomed scorpion sting victims whose clinical picture is dominated by pulmonary oedema, cardiovascular, and many other clinical manifestations. The metabolic, cardio-respiratory changes and electrocardiographic changes are reversed by administration of insulin.

\section{References}

[1] Gueron, M., Margulis, G., Ilia, R. and Sofer, S. (1993) The Management of Scorpion Envenomation 1993. Toxicon, 31, 1071-1076. http://dx.doi.org/10.1016/0041-0101(93)90122-Y

[2] Gueron, M., Adolph, R., Gruff, L., Grup, O., Gabel, M., Grup, G. and Fowler, N.O. (1980) Hemodymamics and Myocardial Consequences of Scorpion Venom. The American Journal of Cardiology, 45, 979-986. http://dx.doi.org/10.1016/0002-9149(80)90166-6

[3] Gueron, M. and Ovsyshcher, I. (1987) What Is the Treatment for the Cardiovascular Manifestations of Scorpion Envenomation? Toxicon, 25, 121-124. http://dx.doi.org/10.1016/0041-0101(87)90170-X

[4] Gueron, M., Marqulis, G. and Sofer, S. (1990) Echocardiographic and Radionuclide Angiographic Observations Following Scorpion Envenomation by Leiurus quinquestriatus. Toxicon, 28, 1005-1009. http://dx.doi.org/10.1016/0041-0101(90)90138-W

[5] Gueron, M., Ilia, R., Shahak, E. and Sofer, S. (1992) Renin and Aldosterone Levels Following Envenomation by the Yellow Scorpion Leiurus quinquestriatus. Toxicon, 30, 765-767. http://dx.doi.org/10.1016/0041-0101(92)90010-3

[6] Ismail, M., Fatani, A.J.Y. and Dabeas, T.T. (1992) Experimental Treatment Protocols for Scorpion Envenomation: A Review of Common Therapies and on Effect of Kallikrein-Kinin Inhibitors. Toxicon, 30, 1257-1279. http://dx.doi.org/10.1016/0041-0101(92)90442-8

[7] Ismail, M. (1993) Serotherapy of the Scorpion Envenoming Syndrome Is Irrationally Convicted without Trial. Toxicon, 31, 1077-1083. http://dx.doi.org/10.1016/0041-0101(93)90123-Z

[8] Ismail, M. (1995) The Scorpion Envenoming Syndrome. Toxicon, 33, 825-858. http://dx.doi.org/10.1016/0041-0101(95)00005-7

[9] La Grange, R.G. (1977) Elevation of Blood Pressure and Plasma Renin Levels by Venom from Scorpions, Centruroides sculpturatus and Leiurus quinquestriatus. Toxicon, 15, 429-433. http://dx.doi.org/10.1016/0041-0101(77)90121-0

[10] Moss, J., Kazie, T., Henry, D.P. and Kopin, I.J. (1973) Scorpion Venom-Induced Discharge of Catecholamines Accompanied by Hypertension. Brain Research, 54, 381-385. http://dx.doi.org/10.1016/0006-8993(73)90065-6

[11] Natu, V.S., Murthy, R.K.K. and Deodhar, K.P. (2006) Efficacy of Species Specific Anti-Scorpion Venom Serum (AScVS) against Severe, Serious Scorpion Sings (Mesobuthus tamulus concanesis, Pocock)—An Experience from Rural Hospital in Western Maharashtra. Journal of the Association of Physicians of India, 54, 283-287.

[12] Amaral, C.F.S., Lopes, J.A., Magalhaes, R.A. and Rezende, N.A. (1991) Electrocardiographic, Enzymatic and Echocardiographic Evidence of Myocardial Damage after Titus Serrulatus Poisoning. The American Journal of Cardiology, 67, 655-657. http://dx.doi.org/10.1016/0002-9149(91)90912-5

[13] Amaral, C.F.S., de Rezende, N.A. and Freire-Maia, L. (1993) Acute Pulmonary Oedema after Tityus serrulatus Scorpion Sting in Children. The American Journal of Cardiology, 71, 242-245. http://dx.doi.org/10.1016/0002-9149(93)90746-Y

[14] Amaral, C.F.S., Barbosa, A.J., Leite, V.H.R., Tafuri, W.L. and de Rezende, N.A. (1994) Scorpion Sting-Induced Pulmonary Oedema: Evidence of Increased Alveolocapillary Membrane Permeability. Toxicon, 32, 999-1003. http://dx.doi.org/10.1016/0041-0101(94)90378-6

[15] Bahloul, M., Rekik, N., Chabchoub, I., Chaari, A., Ksibi, H., et al. (2005) Neurological Complications Secondary to Severe Scorpion Envenomation. Medical Science Monitor, 11, CR196-CR202.

[16] Balasubramaniam, P. and Murthy K.R.K. (1984) Liver Glycogen Depletion in Acute Myocarditis Produced by Scorpion Venom (Buthus tamulus). Indian Heart Journal, 36, 101-103. 
[17] Murthy, K.R.K. and Haghnazari, L. (1999) The Blood Levels of Glucagon, Cortisol and Insulin Following Scorpion (Mesobuthus tamulus concanesis, Pocock) in Dogs. Journal of Venomous Animals and Toxins, 5, 47-55.

[18] Murthy, K.R.K. and Medh, J.D. (1986) Increase in Serum Free Fatty Acids, Phospholipids and Reduction in Total Cholesterol in Acute Myocarditis Produced by Scorpion (Buthus tamulus) Venom Injection in Dogs. Indian Heart Journal, 38, 369-372.

[19] Murthy, K.R.K. and Vakil, A.E. (1988) Elevation of Plasma Angiotensin Levels in Dogs by Indian Red-Scorpion (Buthus tamulus) Venom and Its Reversal by Administration of Insulin and Tolazoline. Indian Journal of Medical Research, 88, 376-379.

[20] Murthy, K.R.K. and Yeolekar, M.E. (1986) ECG Changes in Acute Myocarditis Produced by Scorpion (Buthus tamulus) Venom. Indian Heart Journal, 38, 206-210.

[21] Murthy, K.R.K., Billimoria, F.R., Khopkar, M. and Dave, K.N. (1986) Acute Hyperglycemia and Hyperkalemia in Acute Myocarditis Produced by Scorpion (Buthus tamulus) Venom Injection in Dogs. Indian Heart Journal, 38, 71-74.

[22] Murthy, K.R.K. (1982) Investigations of Cardiac Sarcolemmal ATPase Activity in Rabbits with Acute Myocarditis Produced by Scorpion Venom (Buthus tamulus). Japanese Heart Journal, 23, 835-842. http://dx.doi.org/10.1536/ihj.23.835

[23] Murthy, K.R.K., Hossein, Z., Medh, J.D., Kudalkar, J.A., Yeolekar, M.E., Pandit, S.P. and Billimoria, F.R. (1988) Disseminated Intravascular Coagulation \& Disturbances in Carbohydrate and Fat Metabolism in Acute Myocarditis Produced by Indian Red Scorpion (Buthus tamulus) Venom. Indian Journal of Medical Research, 87, 318-325.

[24] Murthy, K.R.K., Vakil, A.E., Yeolekar, M.E. and Vakil, Y.E. (1988) Reversal of Metabolic and Electrocardiographic Changes Induced by Indian Red Scorpion (Buthus tamulus) Venom by Administration of Insulin, Alpha Blocker and Sodium Bicarbonate. Indian Journal of Medical Research, 88, 450-457.

[25] Murthy, K.R.K., Medh, J.D., Dave, B.N., Vakil, Y.E. and Billimoria, F.R. (1989) Acute Pancreatitis and Reduction of $\mathrm{H}^{+}$Ion Concentration in Gastric Secretions in Experimental Acute Myocarditis Produced by Indian Red Scorpion (Buthus tamulus) Venom. Indian Journal of Experimental Biology, 27, 242-244.

[26] Murthy, K.R.K., Vakil, A.E. and Yeolekar, M.E. (1990) Insulin Administration Reverses the Metabolic and Electrocardiographic Changes Induced by Indian Red Scorpion (Buthus tamulus) Venom in the Experimental Dogs. Indian Heart Journal, 42, 35-42.

[27] Murthy, K.R.K., Shenoi, R., Vaidyanathan, P., Kelkar, K., Sharma, N., Mehta, M., et al. (1991) Insulin Reverses Haemodynamic Changes and Pulmonary Oedema in Children Stung by Indian Red Scorpion Mesobuthus tamulus concanesis, Pocock. Annals of Tropical Medicine and Parasitology, 85, 651-657.

[28] D’Suze, G., Comellas, A., Pesca, L., Sevci, K.C. and Sanchez-De-León, R. (1999) Tityus discrepans Venom Produces a Respiratory Distress Syndrome in Rabbits through an Indirect Mechanism. Toxicon, 37, 173-180. http://dx.doi.org/10.1016/S0041-0101(98)00180-9

[29] Murthy, K.R.K. (2000) The Scorpion Envenoming Syndrome: A Different Perspective. The Physiological Basis of the Role Insulin in Scorpion Envenoming. Journal of Venomous Animals and Toxins, 6, 4-51.

[30] Yugandhar, B., Murthy, K.R.K. and Sattar, S.A. (1999) Insulin Administration in Severe Scorpion Envenoming. Journal of Venomous Animals and Toxins, 5, 200-219.

[31] Bucaretchi, F., Baracat, E.C., Nogueira, R.J., Chaves, A., Zambrone, F.A., et al. (1995) A Comparative Study of Severe Scorpion Envenomation in Children Caused by Tityus bahiensis and Tityus serrulatus. Revista do Instituto de Medicina Tropical de São Paulo, 37, 331-336. http://dx.doi.org/10.1590/S0036-46651995000400008

[32] de Dàvila, C.A.M., Dàvila, D.F., Donis, J.H., de Bellabarba, G.A., Villarreal, V. and Barboza, J.S. (2002) Sympathetic Nervous System Activation, Antivenin Administration and Cardiovascular Manifestations of Scorpion Envenomation. Toxicon, 40, 1339-1346. http://dx.doi.org/10.1016/S0041-0101(02)00145-9

[33] Hering, S.E., Jurca, M., Vichi, F.L., Azevedo-Marques, M.M. and Cupo, P. (1993) "Reversible Cardiomyopathy” in Patients with Severe Scorpion Envenoming by Tityus serrulatus: Evolution of Enzymatic, Electrocardiographic and Echocardiographic Alterations. Annals of Tropical Paediatrics, 13, 173-182.

[34] Sofer, S. and Gueron, M. (1988) Respiratory Failure in Children Following Envenomation by the Scorpion Leiurus quinquestriatus: Hemodynamic and Neurological Aspects. Toxicon, 26, 931-939. http://dx.doi.org/10.1016/0041-0101(88)90258-9

[35] Sofer, S. and Gueron, M. (1992) Cardiovascular Aspects of Scorpion Envenomation. In: Gopalakrishnakone, P. and Tan, C.K., Eds., Recent Advances in Toxinology Research, Venom \& Toxin Research Group, National University of Singapore, Singapore City, 40-49.

[36] Sofer, S., Gueron, M., White, R.M., Lifshitz, M. and Apte, R.N. (1996) Interleukin-6 Release Following Scorpion Sting in Children. Toxicon, 34, 389-392. http://dx.doi.org/10.1016/0041-0101(95)00136-0 
[37] Sofer, S. and Gueron, M. (1988) Respiratory Failure in Children Following Envenomation by the Scorpion Leiurus quinquestriatus: Hemodynamic and Neurological Aspects. Toxicon, 26, 931-939. http://dx.doi.org/10.1016/0041-0101(88)90258-9

[38] Duddin, A.A., Rambaud-Cousson, A., Thalji, A., Juabeh, I.I. and Abu-Libdeh, B. (1991) Scorpion Sting in Children in the Jerusalem Area: A Review of 54 Cases. Annals of Tropical Paediatrics, 11, 217-223.

[39] Gueron, M., Marqulis, G. and Sofer, S. (1990) Echocardiographic and Radionuclide Angiographic Observations Following Scorpion Envenomation by Leiurus quinquestriatus. Toxicon, 28, 1005-1009. http://dx.doi.org/10.1016/0041-0101(90)90138-w

[40] Sofer, S., Shahak, E., Slonim, S. and Gueron, M. (1991) Myocardial Injury without Heart Failure Following Envenomation by the Scorpion Leiurus quinquestriatus in Children. Toxicon, 29, 382-385. http://dx.doi.org/10.1016/0041-0101(91)90293-Z

[41] de Dàvila, C.A.M., Dàvila, D.F., Donis, J.H., de Bellabarba, G.A., Villarreal, V. and Barboza, J.S. (2002) Sympathetic Nervous System Activation, Antivenin Administration and Cardiovascular Manifestations of Scorpion Envenomation. Toxicon, 40, 1339-1346. http://dx.doi.org/10.1016/S0041-0101(02)00145-9

[42] Bahloul, M., Kallel, H., Rekik, N., Ben, H.C., Chelly, H. and Bouaziz, M. (2005) Cardiovascular Dysfunction Following Severe Scorpion Envenomation. Mechanisms and Physiopathology. La Presse Médicale, 34, 115-120.

[43] Tarasiuk, A., Janco, J. and Sofer, S. (1997) Effects of Scorpion Venom on Central and Peripheral Circulatory Response in an Open-Chest Dog Model. Acta Physiologica Scandinavica, 161, 141-149. http://dx.doi.org/10.1046/j.1365-201X.1997.00202.x

[44] Deshpande, S.B. (1988) Indian Red Scorpion (Buthus tamulus) Venom Prolongs Repolarization Time and Refractoriness of the Compound Action Potential of Frog Sciatic Nerve in Vitro. Indian Journal of Experimental Biology, 36, 1108-1113.

[45] Balasubramaniam, P. and Murthy, K.R.K. (1981) Abnormal Cardiovascular and Electrocardiographic Profiles and Cardiac Glycogen Content in Rabbits Injected with Scorpion Venom. Indian Journal of Physiology and Pharmacology, 25, 351-355.

[46] Venkateswaralu, D.Z. and Banu, K.S. (1987) Physiological Effects of Scorpion Venom on Frog's Gastrocnemius Muscle. Indian Journal of Experimental Biology, 13, 429-431.

[47] Achyuthan, K.E., Agarwal, O.P. and Ramachandran, L.K. (1982) Enzymes in the Venoms of Two Species of Indian Scorpions-Heterometrus bengalensis \& Buthus tamulus. Indian Journal of Biochemistry and Biophysics, 19, 356358.

[48] Martin, C. (2004) Scorpion Venom: A Deadly Brew of Toxic Proteins. Biochemistry 4521: Protein Biochemistry.

[49] Chatwal, G.S. and Habermann, E. (1981) Neurotoxins, Protease Inhibitors and Histamine Releasers in the Venom of the Indian Red Scorpion (Buthus tamulus): Isolation and Partial Characterization. Toxicon, 19, 807-823. http://dx.doi.org/10.1016/0041-0101(81)90077-5

[50] Tiwari, A.K. (1992) Isolation, Purification and Functional Characterization of Scorpion Buthus tamulus Venom. M.D. Thesis, Banaras Hindu University, Varanasi.

[51] Hayet, S., Abrough, F. and Djeridane, Y. (2008) Effect and Mechanisms Underlying Scorpion Toxin Action from Androctonus australis garzonii on Atrial Natriuretic Peptide in Rat Atria: An in Vitro Study. Peptides, 29, 364-368. http://dx.doi.org/10.1016/j.peptides.2007.11.019

[52] Harvey, A.L., Anderson, A.J., Brage, M.E.M., Bragal, M.F.M., Marshall, D.L., Rowan, E.G., Vatapour, H., Castneda, O. and Karlson, E. (1992) Toxins Affecting Neuronal Ion Channels. In: Gopalakrishnakone, P. and Tan, C.K., Eds., Recent Advances in Toxinology Research, Venom \& Toxin Research Group, National University of Singapore, Singapore City, 59-70.

[53] Goudet, C., Chi, C.W. and Tytgat, J. (2002) An Overview of Toxins and Genes from the Venom of the Asian Scorpion Buthus martensi Karsch. Toxicon, 40, 1239-1258. http://dx.doi.org/10.1016/S0041-0101(02)00142-3

[54] Borges, A., Alfonzo, M.J., Garćia, C.C., Winand, N.J., Leipold, E. and Heinmann, S.H. (2004) Isolation, Molecular Cloning and Functional Characterization of a Novel Beta-Toxin from the Venezuelan Scorpion, Tityus zulianus. Toxicon, 43, 671-684. http://dx.doi.org/10.1016/j.toxicon.2004.02.022

[55] Shivraj, A., Namoshi, A. and Gao, M.S. (1981) LV Function in Scorpion Sting. Indian Heart Journal, 33, $150-154$.

[56] Posani, L.D., Valdivia, H.H., Ramirez, A.N., Gurrola, G.B. and Martin, B.M. (1992) K ${ }^{+}$Channel Blocking Peptides from the Venom of Scorpions. In: Gopalakrishnakone, P. and Tan, C.K., Eds., Recent Advances in Toxinology Research, Venom \& Toxin Research Group, National University of Singapore, Singapore City, 39-58.

[57] Jouirou, B., Mouhat, S., Andreotti, N., De Waard, M. and Sabatier, J.M. (2004) Toxin Determinants Required for Interaction with Voltage-Gated $\mathrm{K}^{+}$Channels. Toxicon, 43, 909-914. http://dx.doi.org/10.1016/j.toxicon.2004.03.024 
[58] D’Suze, G., Comellas, A., Pesca, L., Sevci, K.C. and Sanchez-De-León, R. (1999) Tityus discrepans Venom Produces a Respiratory Distress Syndrome in Rabbits through an Indirect Mechanism. Toxicon, 37, 173-180. http://dx.doi.org/10.1016/S0041-0101(98)00180-9

[59] Tiwari, A.K. and Deshpande, S.B. (1993) Toxicity of Scorpion (Buthus tamulus) Venom in Mammals Is Influenced by the Age and Species. Toxicon, 31, 1619-1622. http://dx.doi.org/10.1016/0041-0101(93)90346-K

[60] Edwin, K.J., Renin and Angiotensin (2006) Goodman \& Gilman’s The Pharmacological Basis of Therapeutics. 11th Edition, McGraw Hill Medical Publishing Division, New York, 795.

[61] Johnson, D.G., Henry, D.P., Moss, J. and Williams, H.H. (1976) Inhibition of Insulin Secretion Released by Scorpion Toxin on Rat Pancreatic Islets. Diabetes, 25, 198-201. http://dx.doi.org/10.1016/S0041-0101(98)00180-9

[62] Johnson, D.G. and Ensinck, J.W. (1976) Stimulation of Glucagon Secretion by Scorpion Toxin in the Perfused Rat Pancreas. Diabetes, 25, 645-649. http://dx.doi.org/10.2337/diab.25.8.645

[63] Keele, C.A., Neil, E. and Joel, S.N. (2004) Samson Wright’s Applied Physiology. 13th Edition, Oxford University Press, Oxford, 405-512.

[64] Harald, V.M. and Ole, D.M. (1981) Influence of Free Fatty Acids on Myocardial Oxygen Consumption and Ischemic Injury. The American Journal of Cardiology, 48, 361-365. http://dx.doi.org/10.1016/0002-9149(81)90621-4

[65] Devi, S., Reddy, C.N., Devi, S.L., Subramaniam, Y.R., Bhatt, H.V., Suvarnakumari, G., Murthy, D.P. and Reddy, C.R. (1970) Defibrination Syndrome Due to Scorpion Venom Poisoning. British Medical Journal, 1, 345-347. http://dx.doi.org/10.1136/bmj.1.5692.345

[66] Bondy, P.K. and Rosenberg, L.E. (1980) Metabolic Control and Disease. 8th Edition, WB Saunders Co., Phildelphia, 1621.

[67] Fyge, T., Cochran, K.M., Baxter, R.H. and Booth, E.M. (1971) Plasma-Lipid Changes after Myocardial Infarction. Lancet, 298, 997-1001.

[68] Goldstein, R.E., Abumrad, N.N., Lacy, D.B., Wasserman, D.H. and Cherrington, A.D. (1995) Effects of an Acute Increase in Epinephrine and Cortisol on Carbohydrate Metabolism during Insulin Deficiency. Diabetes, 44, 672-681. http://dx.doi.org/10.2337/diab.44.6.672

[69] Oliver, M.F. (1975) The Vulnerable Ischemic Myocardium and Its Metabolism. In: Oliver, M.F., Ed., Modern Trends in Cardiology, 3rd Edition, Butterworths, London, 280-291.

[70] Murthy, K.R.K., Dubey, A.S., Abbas, Z. and Haghnazari, L. (2003) Investigations on the Role of Insulin and Scorpion Antivenom in Scorpion Envenoming Syndrome. Journal of Venomous Animals and Toxins Including Tropical Diseases, 9, 202-238.

[71] Murthy, K.R.K. (2013) Treatment of Scorpion Envenoming Syndrome-Need for Scientific Magnanimity. Indian Medical Association, 111, 254-259.

[72] Scheuer, J. and Stezoski, S.W. (1969) A Protective Effect of Increased Glycogen Stires in Cardiac Anoxia. Journal of Laboratory and Clinical Medicine, 74, 1007-1010. 